\title{
Combining Argo profiles with a general circulation model in the North Atlantic. Part 1: Estimation of hydrographic and circulation anomalies from synthetic profiles, over a year
}

\author{
Gaël Forget ${ }^{a}{ }^{*}$, Bruno Ferron $^{b}$ and Herlé Mercier ${ }^{b}$ \\ ${ }^{a}$ Room 54-1517, Department of Earth, Atmospheric and Planetary Sciences, Massachusetts Institute of \\ Technology, Cambridge, MA 02139, USA \\ ${ }^{\text {b } L a b o r a t o i r e ~ d e ~ P h y s i q u e ~ d e s ~ O c e ́ a n s, ~ U M R ~ 6523, ~ C N R S-I f r e m e r-U B O, ~ I f r e m e r ~ c e n t r e ~ d e ~ B r e s t ~ B . P . ~ 70, ~} 29280$ \\ Plouzané, France \\ *: Corresponding author : G. Forget, email address : gforget@mit.edu
}

\begin{abstract}
:
Argo is a global array of profiling floats that provides temperature $(T)$ and salinity (S) profiles from $2000 \mathrm{~m}$ to the surface every ten days with a nominal spatial resolution of $3^{\circ}$. Here we present idealized experiments where the adjoint method is used to synthesize simulated sets of Argo profiles with a general circulation model, over a one-year period, in the North Atlantic. Using a number of drifting profilers consistent with Argo deployment objectives, the simulated array permits one to identify large-scale anomalies in the hydrography and circulation, despite the presence of a simulated eddy noise of large amplitude. Model dynamics provide an objective means to distinguish eddy noise from large-scale oceanic variability, and to infer the absolute velocity field (including abyssal velocities and sea surface height) from sets of Argo profiles of $\mathrm{T}$ and $\mathrm{S}$. In particular, our idealized experiments suggest that volume and heat transports can be efficiently constrained by sets of Argo profiles. Increasing the number of Argo floats seems to be an adequate strategy to further reduce errors in circulation estimates.
\end{abstract}

Keywords: Data assimilation; 4DVAR; Twin experiment; Argo; General circulation model 


\section{Introduction}

Argo is a global array of profiling floats, which provides temperature $(\mathrm{T})$ and salinity $(\mathrm{S})$ profiles from $2000 \mathrm{~m}$ to the surface every ten days, with a nominal spatial resolution of $3^{\circ}$. This subsurface observing system was designed to complement satellite observations (e.g. of sea surface height) in order to monitor the large-scale and low-frequency oceanic variability (see Roemmich et al., 1999).

One way to investigate the adequacy of Argo as a large-scale observing system is to test whether it permits one to estimate large-scale T and S signals. Argo does not fully resolve the high frequency and small-scale oceanic variability (e.g. meso-scale eddies or internal waves), which thus might be aliased. This suggests the use of state estimation methods that are able to distinguish between large-scale signals of interest and small-scale noise. Guinehut et al. (2002), for example, show that the eddy noise can be efficiently filtered out to estimate monthly $\mathrm{T}$ and $\mathrm{S}$ anomalies in $6^{\circ} \times 6^{\circ}$ squares, using objective analysis (Bretherton et al., 1976). The adequacy of Argo as a large-scale observing system can also be investigated with respect to large-scale circulation fields besides $\mathrm{T}$ and $\mathrm{S}$. As each pair of profiles can be interpreted as a direct measurement of the geostrophic shear, Argo can be expected to significantly improve our knowledge of the large-scale circulation through the use of inverse methods. Unlike previous studies, the adequacy of Argo is here investigated in terms of both the hydrography (i.e. $\mathrm{T}$ and $\mathrm{S}$ ) and the circulation, with an emphasis on the latter.

The estimation method employed here, known as the "adjoint method" or the "4DVAR assimilation" (see e.g. Wunsch, 1996, and Bennett, 2002), consists of searching for the solution of a general circulation model (GCM) that best fits the observations in a least-squares sense, over a time interval. In this context, a GCM can be interpreted as a covariance operator, which accounts for a variety of length scales (from the model grid-scale, up to the model domain size), for a variety of time scales (from the model time-step, up to the simulated time interval), for the dynamical relationships between the different physical quantities, and for the anisotropy associated with oceanic structures. In particular, the GCM-based interpolation tool can be regarded as a non-linear inverse model in which the velocity field is estimated along with the tracer fields. The GCM-based interpolation framework is therefore appropriate to investigate the adequacy of Argo as a large-scale observing system as a whole, i.e. with respect to both the hydrography and the circulation. 
We want to provide insights into the two following problems: by processing sets of Argo profiles with GCM-based interpolation, can the large-scale T and S signals be distinguished from the small-scale noise? To what extent can the large-scale circulation be inverted from sets of Argo ( $\mathrm{T}$ and $\mathrm{S}$ ) profiles?

The complexity of the problem argues for a two-step approach. Indeed, assume that only real-observation experiments are carried out, and the resulting Argo-GCM estimates $\left(X_{e}\right.$, for a quantity $X)$ are evaluated based on independent information $\left(X_{i}\right) . X_{e}$ could be a poor estimate of $X_{i}$ if the information content of sets of Argo profiles was insufficient: (issue A) $X$ may be dominated by scales that are too small compared with the Argo sampling; (issue B) if $X$ is velocity information, it simply may not be possible to determine $X$ uniquely from sets of T and S profiles. But, in practice, $X_{e}$ could also be a poor estimate of $X_{i}$ if the estimation system had faults: (issue $\mathrm{C}$ ) the least-squares solution $\left(X_{e}\right)$ may be incorrect because of improper assumptions on error statistics; (issue D) errors in the GCM dynamics may prevent the adequate fit to Argo profiles. If $X_{e}$ was a poor estimate of $X_{i}$, it would then be unlikely that a clear explanation emerges. Now, idealized experiments using GCM simulated observations permit one to investigate issues $\mathrm{A}$ and $\mathrm{B}$ alone, leaving issues $\mathrm{C}$ and $\mathrm{D}$ as the main concerns when assimilating real observations. A two-step approach is therefore used: first an idealized context is considered where data noise statistics and GCM dynamics are assumed perfect (here), and then the case of real-observations is treated (companion paper, Forget et al., 2007).

Also, idealized experiments provide unique opportunities to investigate issues A and B. Indeed, the time-varying GCM state to be estimated (formally: the truth) is known completely and perfectly in idealized experiments, in contrast to the case of real oceans when $X_{i}$ can be fairly uncertain, incomplete or simply non-existent. For example, idealized experiments allow one to test the possibility of inferring anomalies of volume and tracer transports from Argo measurements (Section 3). Furthermore, the data set characteristics are fully adjustable parameters in the idealized framework, and this permits a discussion of observing system design. For example, one can test whether increasing the number of Argo floats could significantly improve circulation estimates (Section 4). 


\section{Experiment configurations}

\subsection{Estimation problem}

Consider an estimation problem that consists of finding a set of model parameters, $x$, that yields a least-squares fit of the model trajectory to noisy data, $y_{o}$, and to an uncertain prior estimate of $x, x_{b}$. The model counterpart to $y_{o}, y(x)$, is a function of $x$. This problem can be solved by minimizing a cost function $J(x)$ that, following Ide et al. (1997), is written as :

$$
J(x)=J_{b}+J_{o}=\left(x-x_{b}\right)^{T} B^{-1}\left(x-x_{b}\right)+\left(y(x)-y_{o}\right)^{T} R^{-1}\left(y(x)-y_{o}\right)
$$

where the weighting matrices $B^{-1}$ and $R^{-1}$ are the inverses of error covariance matrices. In practice, $J$ is minimized iteratively using descent directions computed by the adjoint of the model (Marotzke et al., 1999), and an optimization algorithm (Gilbert and Lemaréchal, 1989). Below, this process is referred to as assimilation. For this study, $x$ are the model initial conditions of temperature and salinity, and the optimization starts from $x=x_{b}$.

The data term $J_{o}$ measures the distance between the data $y_{o}$ and their model counterparts $y$. The data are a set of $\mathrm{T}$ and $\mathrm{S}$ profiles, which are distributed in space and over a period that, below, is referred to as the assimilation window. The assimilation window is chosen as one year, which is an intermediate value for this parameter (e.g. between the value of six years used by Stammer et al. 2002 and the value of one month used by Weaver et al. 2003).

The assimilation window choice can be expected to have fundamental consequences. Over one year, with the exception of low latitudes (see Gill and Niiler, 1973), the large-scale baroclinic circulation is not expected to strongly evolve and is thus mainly controlled by the initial state. Furthermore, the large-scale circulation is expected to adjust geostrophically to the initial conditions of $\mathrm{T}$ and $\mathrm{S}$ with a time scale much shorter than one year. Therefore, for a one-year assimilation window, it is reasonable to focus on the optimization of the $\mathrm{T}$ and $\mathrm{S}$ initial conditions.

Also, the information is expected to propagate farther through GCM dynamics (e.g. due to advection and waves) as the assimilation window increases. The assimilation window 
choice can therefore be regarded as a compromise between propagating the observational constraint (desirable) and propagating model error (undesirable). The latter is only relevant in the case of real data, since model dynamics are assumed perfect in the idealized experiments, so further discussion is provided in the companion paper (Forget et al., 2007).

\subsection{Model set up}

The model is a North Atlantic (from $20^{\circ} \mathrm{S}$ to $70^{\circ} \mathrm{N}$ ) configuration of the MITgcm (Marshall et al., 1997). It has 23 levels with a refined vertical resolution near the surface, and a $1^{\circ} \cos$ (latitude) horizontal resolution. The low model resolution is chosen for computational practicality. Similar to most large-scale state estimation studies (e.g. Stammer et al., 2002, and Ferron and Marotzke, 2003), the model does not resolve eddies. The vertical and horizontal viscosities are $10^{-3} \mathrm{~m}^{2} \mathrm{~s}^{-1}$ and $2 \times 10^{4} \mathrm{~m}^{2} \mathrm{~s}^{-1}$ respectively. For $\mathrm{T}$ and $\mathrm{S}$, the vertical and horizontal diffusivities are $10^{-5} \mathrm{~m}^{2} \mathrm{~s}^{-1}$ and $10^{3} \mathrm{~m}^{2} \mathrm{~s}^{-1}$ respectively. Lateral noslip and bottom free-slip boundary conditions are used. A convective adjustment is applied in statically unstable cases. The adjoint of the MITgcm is automatically generated with the Tangent linear and Adjoint Model Compiler (TAMC, Giering and Kaminski, 1998).

The model is forced with daily heat, freshwater, and momentum fluxes from the 19791993 ERA15 ECMWF reanalysis. A relaxation of surface temperature and salinity to the Reynaud et al. (1998) seasonal climatology is added to the forcing, with a time scale of 30 days. This climatology is also used to define the $\mathrm{T}$ and $\mathrm{S}$ initial conditions for the spin-up and to prescribe buffer zones along the artificial walls at $20^{\circ} \mathrm{S}, 70^{\circ} \mathrm{N}$, and at $14^{\circ} \mathrm{E}$ in the Mediterranean Sea. A model spin-up is first performed starting from summer 1979 to summer 1987. Early in the spin-up, the model undergoes a significant drift away from the climatological initial state. In the later stages of the spin-up, the model exhibits low-frequency evolutions of smaller amplitude, about a state that is presumably more consistent with the GCM dynamics than is the Reynaud et al. (1998) climatology.

The model solution for summer 1987-summer 1988 is hereafter referred to as the reference integration. In our idealized framework, it represents a true oceanic 4D state that has to be estimated. Starting from an integration with perturbed initial conditions in summer 1987, observations generated from the reference integration are assimilated in an attempt to estimate the reference integration. 


\subsection{Initial condition perturbations}

The perturbed initial conditions in summer $1987\left(x_{b}\right)$ are chosen as some instantaneous T and S model fields of summer 1989. The other model parameters (e.g. the initial velocity field, the forcing, etc) are those of the reference integration. Note that the heat and freshwater surface fluxes can still change through the relaxation terms when the model trajectory is modified, as a consequence of modifications in the initial conditions. The model integration from $x_{b}$ in summer 1987 to summer 1988 is the first guess trajectory of the estimation process.

$J_{b}$, from Eq. 1, is written as $\Sigma_{i, j, k}\left(x(i, j, k)-x_{b}(i, j, k)\right)^{2} / \sigma_{b}^{2}(i, j, k)$, where $(i, j, k)$ are grid point indices corresponding to longitude, latitude and depth respectively. Such a diagonal approximation of the covariance matrix $B$ is common practice (e.g. Stammer et al., 2002, and Ferron and Marotzke, 2003) as it saves much numerical storage. Here $\sigma_{b}(i, j, k)$ is further approximated as $\sigma_{b}(k)$, which is computed at each level $\mathrm{k}$ as the root mean squared (RMS) misfit between $x_{b}$ and the initial conditions of the reference integration. Fig. 1 shows the $\sigma_{b}(k)$ profiles for $\mathrm{T}$ and $\mathrm{S}$ in the main experiment (hereafter ARGOlike). Fig. 1 also shows the $\sigma_{o}(k)$ data error profiles discussed in the next section.

Below, unless otherwise specified, we reserve the words signal for the difference between the first guess and reference integrations, residual for the difference between the optimal (i.e. after assimilation) and reference integrations, and correction for the difference between the optimal and first guess integrations. Hence, the correction must be the opposite of the signal to obtain a perfect estimate with zero residual. These conventions are summarized in Table 1. By design, the signal mimics oceanic interannual anomalies and is fully consistent with the GCM dynamics; it is mostly large-scale and low-frequency, as will be illustrated in Section 3.

\subsection{Data set simulation}

Synthetic T and S profiles are generated from the reference integration in the area off the $2000 \mathrm{~m}$ isobath that can be observed by Argo floats (Fig. 2). For experiment ARGOlike, the positions of 300 floats are first randomly initialized. This number of floats is consistent with the Argo array nominal characteristics (see Roemmich et al., 1999). The floats are 
then advected once a day at $850 \mathrm{~m}$ and profile simultaneously every ten days from 2450 m up to the surface. Fig. 2 illustrates the drift of the array in ARGOlike.

When assimilating real data, the estimate of data errors must account for at least two error sources: instrumental errors and representation errors. The latter are the inconsistencies between the model and the ocean physics that are regarded as unavoidable misfits. As oceans are highly turbulent (see e.g. Stammer, 1997), the overall errors are expected to be large for a $1^{\circ}$ resolution model that does not resolve turbulence (even if measurements are very accurate) owing to representation error. To make the idealized framework more relevant to the assimilation of real data, a noise of large amplitude has to be added to the synthetic data.

The additional noise is Gaussian, uncorrelated both in space and in time, and its amplitude, $\sigma_{o}(k)$, only depends on depth. For such a noise, $R(i, j, k)=\sigma_{o}^{2}(k)$ provides an exact least square formulation for $J_{o} . \sigma_{o}(k)$ in experiment ARGOlike (Fig. 1) is chosen as the median value of the $1^{\circ}$ resolution standard deviation fields associated with the Reynaud et al. (1998) climatology, which provides an estimate of oceanic small-scale noise. The noise to signal ratio $(N / S)$ is defined as the average of $\sigma_{o}(k) / \sigma_{\text {sig }}(k)$ over $\mathrm{k}$, where $\sigma_{\text {sig }}(k)$ is the RMS monthly mean signal at level k. $N / S$ is a key control parameter of the idealized estimation problem, as opposed to the numerical values of $\sigma_{o}(k)$ and $\sigma_{b}(k)$ separately. $N / S$ is 1.45 in ARGOlike, which thus involves a data noise of relatively large amplitude as can be expected to occur in real-observations assimilation. Section 4 discusses experiments (see Table 2) with other values of $N / S$.

Below, $j_{o}^{\text {res }}(t)$ and $j_{o}^{\text {sig }}(t)$ denote the average of $J_{o}$ at observation time $t$, after and before assimilation respectively. Also, $j_{\text {perfect }}$ denotes the analog of $j_{o}$ for the perfect observations (i.e. the ones without the added noise). Let us stress that the perfect observations are never assimilated, but $j_{\text {perfect }}$ is used to discuss the assimilation results. Both $j_{o}$ and $j_{\text {perfect }}$ are normalized mean squared model-data misfits. 


\section{Results of the ARGOlike experiment}

\subsection{Model fit to the data}

Consistent with least-squares estimation theory, $j_{o}^{\text {res }}$ is on average close to 1 (Fig. 3, left), reflecting that the amplitude of model-data misfits after assimilation is close to the (perfectly known) noise level. Moreover, $j_{o}^{\text {res }}$ does not show any significant temporal evolution, consistent with the absence of model error accumulation here. The reduction of $j_{o}$ by the assimilation (Fig. 3, left) could result from a fit to data noise (e.g. see Thacker and Long, 1988). However, beyond the first two months, $j_{o}$ and $j_{\text {perfect }}$ (Fig. 3, right) are similarly reduced by the assimilation. This contradicts the data noise fit hypothesis, since signal and data noise are uncorrelated here. Some fit to data noise seems to occur in the first two months though, when $j_{o}^{r e s}<j_{\text {perfect }}^{\text {res }}+1$. The reduction of $j_{\text {perfect }}$ by a factor of ten demonstrates that the assimilation brings the model significantly closer to the reference integration.

\subsection{Improvements in $T$ and $S$ fields}

The mean squared misfits to the reference integration fields are now considered (Fig. 4), which actually measure the quality of the large-scale estimates. First, the signals (Fig. 4) slowly decrease with time, as can be expected for a diffusive model when no active forcing anomalies are introduced. The decrease is stronger in the upper $100 \mathrm{~m}$ due to the surface relaxation terms (Fig. 4), and this explains most of the temporal decrease in $j_{o}^{s i g}$ and $j_{\text {perfect }}^{\text {sig }}$ (Fig. 3). Second, in the observed layers $(0-2450 \mathrm{~m})$, the mean squared residuals are significantly smaller than the mean squared signals (Fig. 4), except in the first two months (see below). The maximum in residuals at $600 \mathrm{~m}$ (Fig. 4) does not reflect a general behavior but is specific to this experiment, as shown in Section 4. Over the last six months (Table 3) the mean squared residuals are $70 \%$ smaller than the mean squared signals; this is consistent with the range of error variance reduction reported by Guinehut et al. (2002) (their Tables 1 and 2). Third, in the unobserved layers (below $2450 \mathrm{~m}$ ) residuals for $\mathrm{T}$ and $\mathrm{S}$ are barely smaller than the corresponding signals (Fig. 4). Though

model dynamics may further propagate the upper-ocean information into the deep ocean for a longer assimilation window, this suggests the need for direct observations to improve 
the model deep hydrography over a year.

Two 2D sections are now considered (Figs. 5 and 6 ). Those illustrate that the $\mathrm{T}$ signals (panels A) are dominantly large-scale and low-frequency. The same is true for the $\mathrm{S}$ signals and the circulation signals (not shown). To be more quantitative, we compute largest-scales contributions (defined as the average over $5^{\circ} \times 5^{\circ}$ boxes) and intermediatescales contributions (defined as the remainder) to signals and residuals. The mean squared signal is $90 \%$ largest-scales. The improvement in $\mathrm{T}$ and $\mathrm{S}$ fields due to the assimilation (Fig. 4, Table 3) is mostly a reduction of large-scale misfits to the reference integration (Figs. 5B and 6B). For intermediate-scales contributions, the mean squared residual is $50 \%$ smaller than the mean squared signal. The corrections applied to the initial conditions grow towards large-scales and low-frequencies over the first two months (Fig. 6, C and D), as a result of the integration of GCM dynamics.

As a conclusion, GCM dynamics provide, by themselves, an efficient means to interpolate $\mathrm{T}$ and S fields from sets of Argo profiles. The use of GCM dynamics permits one to reveal the large-scale information (present section) and filter out the small-scale, large amplitude, data noise (previous section).

\subsection{Improvements in the circulation}

The possibility of inverting large-scale low-frequency circulation anomalies from sets of Argo profiles, which is our main motivation for using GCM-based interpolation, is now investigated. The results are discouraging for the first two months of integration: the mean squared velocity misfits to the reference integration (Fig. 7) are larger after assimilation than before. During this period, dynamical adjustments to unbalanced initial condition perturbations take place, and these are associated with an excess of kinetic energy (not shown). Along with the results for $\mathrm{T}$ and $\mathrm{S}$, the solution over the first months is thus found useless and will not be further analyzed. But after only three months of integration, the impact of the assimilation on the velocity field becomes positive. The reductions in mean squared misfits to the reference integration due to the assimilation that are reported below (incl. numbers from Tables 3 and 4) are computed using monthly means over the last six months. The mean squared residuals are $65 \%$ smaller than the mean squared signals for velocities (Table 3; also see Fig. 7), and this reduction in velocity misfits reflects that 
large-scale velocity signals have been successfully estimated (Fig. 8). At the sea surface, the improvements in the velocity field translate, as expected, into improvements in the dynamic topography (Fig. 9). Using GCM-based interpolation, we thus show that Argo profile information (no velocity observation is assimilated) is as valuable with respect to the absolute velocity field as it is with respect to $\mathrm{T}$ and $\mathrm{S}$ fields (Table 3).

The next question to be addressed is the nature of the improvements in the circulation associated with the velocity misfit decrease. If we decompose velocity anomalies into barotropic and baroclinic components (i.e. the vertical average and the rest respectively), we find that mean squared residuals are about $65 \%$ smaller than the mean squared signals for both components. It is remarkable that the absolute velocity field is significantly improved even though the deep hydrography is barely improved. The deep hydrography does not dominate the flow structure here. For the deep velocities themselves, the mean squared residual is 55\% smaller than the mean squared signal (only $30 \%$ for the deep hydrography). Thus, an upper-ocean Argo set of T and S profiles can efficiently constrain both barotropic and baroclinic components of the velocity field.

Now we turn our attention to volume and heat transports, which are of particular interest for climate research. Volume transports are analyzed in terms of barotropic streamfunction $(\Psi)$ and overturning streamfunction $(\Phi)$, defined as

$$
\begin{aligned}
\Psi\left(i_{0}, j_{0}\right) & =\sum_{1}^{i_{0}} \sum_{1}^{k_{\max }} v\left(i, j_{0}, k\right) \times a\left(i, j_{0}, k\right) \\
\Phi\left(j_{0}, k_{0}\right) & =\sum_{1}^{i_{\max }} \sum_{1}^{k_{0}} v\left(i, j_{0}, k\right) \times a\left(i, j_{0}, k\right)
\end{aligned}
$$

where $v$ is the meridional velocity, and $a$ is the associated model cell surface. For $\Psi$, the mean squared residual is $72 \%$ smaller than the mean squared signal. This improvement in $\Psi$ (Fig. 10, upper panels) reflects a basin-scale improvement in the barotropic component of the velocity. Relationships between barotropic volume transports and hydrography have been derived in a theoretical framework (e.g. Greatbatch et al., 1991, and Mertz and Wright, 1992). We simply find that, in practice, sets of Argo profiles can provide an efficient constraint of the barotropic volume transports. From a methodological point of view, we show that GCM-based interpolation is a convenient way to diagnose the barotropic streamfunction from a set of noisy profiles distributed in space and time. The same conclusions hold for $\Phi$ (Fig. 10, lower panels), for which the mean squared residual 
is $77 \%$ smaller than the mean squared signal. The overturning streamfunction measures basin-scale vertical volume transports; its relationship with hydrography in the presence of complex topography remains unclear. Our result argues for the following relationship: within GCM dynamics, in the presence of complex topography, a constraint of just the hydrography implies a constraint of basin-scale vertical volume transports. We show that Argo can provide the bulk of the information needed to diagnose basin-scale vertical volume transports.

Heat transports are analyzed in terms of the advective meridional heat transport $(\Theta$, Eq. $4)$, and of its so-called overturning component $\left(\Theta_{o v}\right.$, Eq. 5) and gyre component $\left(\Theta_{g y}\right.$, Eq. $6)$, defined as

$$
\begin{aligned}
\Theta\left(j_{0}\right) & =\rho_{0} C_{p} \sum_{1}^{i_{\max }} \sum_{1}^{k_{\max }} v\left(i, j_{0}, k\right) \times T\left(i, j_{0}, k\right) \times a\left(i, j_{0}, k\right) \\
\Theta_{o v}\left(j_{0}\right) & =\rho_{0} C_{p} \sum_{1}^{i_{\max }} \sum_{1}^{k_{\max }} \bar{v}\left(j_{0}, k\right) \times \bar{T}\left(j_{0}, k\right) \times a\left(i, j_{0}, k\right) \\
\Theta_{g y}\left(j_{0}\right) & =\rho_{0} C_{p} \sum_{1}^{i_{\max }} \sum_{1}^{k_{\max }}\left(v\left(i, j_{0}, k\right)-\bar{v}\left(j_{0}, k\right)\right) \times\left(T\left(i, j_{0}, k\right)-\bar{T}\left(j_{0}, k\right)\right) \times a\left(i, j_{0}, k\right)
\end{aligned}
$$

where the overbar denotes a zonal average, $\rho_{0}$ is $1000 \mathrm{~kg} \mathrm{~m}^{-3}$, and $C_{p}$ is $4000 \mathrm{~m}^{2} \mathrm{~kg} \mathrm{~s}^{-2}$. Note that this decomposition of $\Theta$ is not exact, as reflected by Fig. 11 (upper right). The mean squared residuals are $67 \%, 60 \%$, and $81 \%$ smaller than the mean squared signals for $\Theta, \Theta_{o v}$, and $\Theta_{g y}$ respectively. The improvement in $\Theta$ (Fig. 11, upper left) here mainly results from an improvement in $\Theta_{o v}$ (Fig. 11, lower left), since the signal in $\Theta_{o v}$ dominates the signal in $\Theta$ (except at high latitudes). It not necessary that improvements in $\Theta$ are associated with improvements in volume transports, because $\Theta$ is a product of velocity and temperature fields. Improvements in volume transports are crucial in this experiment though: if we neglect them and only account for the change in temperature fields due to the assimilation, then improvements in $\Theta$ are much smaller (not shown). As a conclusion, we find that sets of Argo profiles can significantly improve estimates of volume and heat transports; this result is very promising as the direct observation of such quantities remains a challenge.

We conclude this section with two comments on the above results. First, the perspective of our study is not to understand the driving forces of the circulation, but rather to investigate the possibility of diagnosing it using modern observing systems (here Argo 
profiles). Second, some of the hypotheses of our study may limit the relevance of the above results, and they should eventually be re-examined. For example: 1) a more general problem could include active surface forcing perturbations along with initial condition perturbations; 2) the present model does not resolve turbulence that may play a significant role for heat transport. In general, the efficiency of the basin-scale circulation constraint provided by Argo profiles might depend on the particular GCM and estimation method.

\section{Sensitivity experiments}

The sensitivity of the estimates' quality to the data set characteristics is discussed here; the sensitivity experiments' set-up is summarized in Table 2. Exp. moreNOISE addresses an even noisier ocean. Exp. lessNOISE addresses an ocean with relatively little data noise. In exp. EULERIAN, instruments' position remain constant, to simulate a moored array. In exp. RANDOM, instruments' position are chosen randomly every ten days, to test whether an underestimation in floats dispersion in ARGOlike (a likely case as the GCM circulation used to advect floats does not include turbulence) may affect the results. In experiments signal1, signal2 and signal3, we use three different perturbations of initial conditions, which result in three different realizations of the signal. In these three experiments, the sampling is the same as in ARGOlike, and $N / S$ ranges between ARGOlike's values and moreNOISE's values (Table 2). Each of the sensitivity experiments consists of 20 optimization iterations, bringing $j_{o}^{r e s}$ close to unity, consistent with ARGOlike.

As expected, estimates of $\mathrm{T}$ and $\mathrm{S}$ improve when the number of floats increases or the data noise decreases (Table 3), as it does when using objective analysis (Guinehut et al., 2002, their Tables 1 and 2). In the most noisy ocean (exp. moreNOISE), mean squared signals only represent $16 \%\left(=100 /\left(N^{2} / S^{2}\right)\right)$ of the noise variance, but mean squared residuals are still $63 \%$ smaller than mean squared signals. This confirms that the GCM-interpolation tool efficiently distinguishes between large-scale signal and small-scale noise. If only 100 floats are used (exp. fewerFLOATS), patches of residual are found in the wide areas that are deprived of observations (Fig. 5C). If 900 profilers are used (exp. moreFLOATS), the residuals' amplitude is fairly homogeneous in space (Fig. 5 D), the exception being shallow regions that remain deprived of observations and show patches of residual. In this experiment, mean squared residuals are $75 \%$ smaller than mean squared signals for the intermediate-scales (defined in Section 3.2). Estimates' quality is very similar in ARGOlike 
and EULERIAN (Table 3), showing that the slow drift of the array due to the lowresolution model circulation has little impact over one year. The simulated increase in floats dispersion (from exp. ARGOlike to exp. RANDOM) has a more significant impact: it improves the estimates' quality nearly as much as dividing the data noise level by 5 (exp. lessNOISE) or multiplying the number of floats by 3 (exp. moreFLOATS). Our interpretation of this result is that sampling randomly maximizes the independence of observations. If turbulence did increase both data noise level and floats dispersion, this result suggests that the two effects might compete in terms of large-scale estimates' quality. RANDOM should be regarded as an extreme dispersion case though, and it is not clear whether turbulent dispersion can have a significant impact in reality.

Estimates of $\mathrm{U}$ and $\mathrm{V}$ get improved or degraded along with estimates of $\mathrm{T}$ and $\mathrm{S}$ (Table 3) - improving observational constraints for the hydrography has a direct impact on the circulation estimate's quality. In general, such a behavior requires that hydrography contains the bulk of the information on the absolute velocity field. We find that multiplying the number of Argo floats by a factor of 3 (exp. moreFLOATS) compared with the array nominal density (exp. ARGOlike) can reduce mean squared errors in the velocity field by a factor of 2 (Table 3). The same conclusions hold for volume and heat transports as well; mean squared residuals are $85 \%, 85 \%$, and $90 \%$ smaller than mean squared signals for $\Phi, \Psi$ and $\Theta$ (Fig. 11) respectively in moreFLOATS (77\%, $72 \%$ and $67 \%$ in ARGOlike). Increasing the number of Argo floats thus seems to be an adequate observational strategy as far as improving circulation estimates is concerned.

Improvements in the hydrography and in the velocity field in exp. signal1, signal2, signal3, ARGOlike, and moreNOISE are fairly similar to one another (Table 4). The above results thus do not seem to correspond to an abnormally unfavorable or abnormally favorable realization of the signal. The $N / S$ ratio varies only slightly among the experiments of Table 4 , and performances scattering among those experiments partly reflects that observations and strong signals can be collocated or otherwise. For example, the maximum in residuals at $600 \mathrm{~m}$ for $\mathrm{T}$ and $\mathrm{S}$ in ARGOlike (Fig. 4) does not occur in signal1, signal2 and signal3 (not shown). This maximum in ARGOlike results from unobserved large signals in shallow regions. Also, we have slightly modified the assimilation problem set-up in signal1, signal2 and signal3 compared with the other experiments: a) initial conditions of velocity are perturbed along with the $\mathrm{T}$ and $\mathrm{S}$ initial conditions, whereas $x$ is still restricted to $\mathrm{T}$ and $\mathrm{S} ; \mathrm{b}$ ) the forcing is augmented with the fluxes due to the relaxation terms that were 
computed during the reference integration, and the relaxation terms are then turned off during the assimilation process. As expected, these details of the set-up do not have major effects on the results (Table 4).

\section{Summary and discussion}

A series of idealized assimilation experiments have been conducted, over a one year period, in the North Atlantic. Large-scale anomalies that reflect the GCM low-frequency variability are estimated from sets of noisy synthetic profiles of $\mathrm{T}$ and $\mathrm{S}$ mimicking Argo observations. The number of simulated drifting floats is consistent with Argo objectives. The data noise amplitude is chosen as large as the amplitude of the large-scale anomalies, which is relevant to the case of real observations where eddy signals can induce large representation errors. The results suggest that the design of Argo is consistent with largescale state estimation objective as it permits one to identify large-scale anomalies of the hydrography and of the circulation despite large-amplitude small-scale noise.

The set of pointwise and noisy profile constraints yields a reduction by $70 \%$ of the mean squared misfits to the true $\mathrm{T}$ and $\mathrm{S}$ fields in the upper-ocean observed layers (except in the first months). This is in good agreement with the results of Guinehut et al. (2002) who used objective analysis - with respect to the hydrography, the most novel aspect of this study is the use of GCM-based interpolation. It is shown that integrating GCM dynamics over a year, by itself, permits one to distinguish between large-scale signals and small-scale noise, and allows the projection of the pointwise information onto large-scales. Over the one-year integration period considered, information from the upper-ocean Argo profile constraints do not propagate deep enough to significantly improve the hydrography below the observed layers.

Beyond the estimation of the hydrography, the primary novelty of this study is that we have shown the Argo program to be very useful to monitor low-frequency variations in the circulation. Indeed, with the exception of the first months, the upper-ocean profile constraints yield a reduction of mean squared velocity errors by $65 \%$ in the upper-ocean (55\% in the deep unobserved layers). Noteworthy, this impact is on the absolute velocities, including both baroclinic and barotropic components. Of particular interest for climate research, we find that Argo can provide an efficient constraint on volume and heat trans- 
ports. Thanks to the upper-ocean profile constraints, the mean squared errors in overturning streamfunction, in barotropic streamfunction, and in meridional heat transport are reduced by $77 \%, 72 \%$ and $67 \%$ respectively. From a methodological standpoint, the results illustrate that GCM-based interpolation provides an efficient tool to interpret a set of noisy pointwise Argo profiles in terms of the large-scale circulation (incl. the basin-scale circulation), at least for simulated observations.

This appealing behavior of the GCM estimation system requires a long enough time of integration: in the first months, while the model trajectory correction grows towards large-scales, $\mathrm{T}$ and $\mathrm{S}$ fields are almost unimproved by the assimilation, the velocity field is even degraded by the assimilation, and some fit to data noise occurs. In the companion paper (Forget et al., 2007), this leads us to not use first months solutions. A more elegant strategy, which is beyond the scope of our study, would be useful to save computational cost. It is remarkable, however, that only three months of integration are necessary for circulation estimates to become useful - not years. It is also noticeable that $\mathrm{T}$ and $\mathrm{S}$ estimates can become useful even faster than circulation estimates.

The large-scale information provided by Argo depending on the array characteristics has been further investigated through a series of sensitivity experiments. As expected (for T and $\mathrm{S}$ at least), estimates improve when the number of floats increases or the data noise decreases. If the number of floats is significantly smaller than Argo objectives, large-scale error patches appear in areas that are deprived of observations. Mean squared errors are reduced $63 \%$ by the profile constraints even when mean squared signals only represent $16 \%$ of the noise variance. It is suggested that turbulent dispersion of Argo floats can be helpful to large-scale state estimation by increasing independence of observations. In general, the circulation estimate's quality varies along with estimates' quality for $\mathrm{T}$ and S. Errors in circulation estimates get reduced by a factor of 2 when we multiply the Argo nominal number of floats by 3 ; we thus conclude that increasing the number of Argo floats seems an adequate observational strategy as far as improving circulation estimates is concerned.

These results are encouraging in the perspective of real observations: the present idealized experiments may be viewed as an attempt to prove Argo inadequate as a large-scale lowfrequency observing system, and this attempt has failed. Extrapolating the results to the case of real observations requires caution though. Crucially, the simulated observations 
are perfectly consistent with the GCM dynamics in this study, and this is no longer true for sets of real Argo profiles. In the worst case scenario, errors in the GCM dynamics may preclude any useful skill of the GCM interpolation/inversion tool for real Argo profiles. This demands an investigation that will be specific to the GCM used, and the companion paper (Forget et al., 2007) is such a study for the present GCM configuration.

Other possible perspectives of the present study include a baseline for further idealized experiments. For example: non-diagonal multivariate covariance matrices (e.g. see Weaver and Courtier, 2001) could be used to try to improve the estimation system behavior in the first months; a more realistic representation of turbulent effects (on data noise, on floats' advection, and on the estimation problem size) could be considered; details of the floats behavior (maximum observation depth, drift depth, drift during surface emission, etc.) could be refined, and may deserve discussion on a regionial basis (e.g. see Schiller et al. 2004 for the Indian Ocean); the results could be put in the perspective of other observing systems (altimeters in particular); more emphasis could be put on the seasonal cycle, and, more generally, on intra-annual variations, by including forcing errors in the estimation problem. In eddy resolving ocean state estimation, our results suggest that Argo would efficiently constrain the large-scale circulation, but this still has to be assessed in practice.

\section{Acknowledgments}

Numerical experiments used the NEC SX-5 of the IDRIS (Orsay, France) (project \#031532). Bruno Ferron and Herlé Mercier are supported by CNRS, Gaël Forget by DGA. Additional support was provided by the Groupe Mission Mercator Coriolis. 


\section{References}

Bennett, A., 2002. Inverse modeling of the ocean and atmosphere. Cambridge University Press.

Bretherton, F., Davis, R., Fandry, C., 1976. A technique for objective analysis and design of oceanographic experiments applied to MODE-73. Deep-Sea Res. 23, 559-582.

Ferron, B., Marotzke, J., 2003. Impact of 4d-variational assimilation of WOCE hydrography on the meridional circulation of the Indian Ocean. Deep-Sea Res. 50, 2005-2021.

Forget, G., Mercier, H., Ferron, B., 2007. Combining ARGO profiles with a general circulation model in the North Atlantic. part II: realistic tranports and improved hydrography, between spring 2002 and spring 2003. OcMo submitted.

Giering, R., Kaminski, T., 1998. Recipes for adjoint code construction. Trans. Math. Soft. 24.

Gilbert, J., Lemaréchal, C., 1989. Some numerical experiments with variable-storage quasiNewton algorithms. Mathematical Programming 45, 407-435.

Gill, A., Niiler, P., 1973. The theory of seasonal variability of the ocean. Deep-Sea Res. $20,141-177$.

Greatbatch, R., Fanning, A., Goulding, A., Levitus, S., 1991. A diagnosis of interpentadal circulation changes in the North Atlantic. J. Geophys. Res. 96, 22009-22023.

Guinehut, S., Larnicol, G., Le Traon, P., 2002. Design of an array of profiling floats in the North Atlantic from model simulations. J. Mar. Syst. 35, 1-9.

Ide, K., Courtier, P., Ghil, M., Lorenc, A., 1997. Unified notation for data assimilation : operational, sequential and variational. J. Meteo. Soc. Jpn. 75, 181-189.

Marotzke, J., Giering, R., Zhang, Q. K., Stammer, D., Hill, C. N., Lee, T., 1999. Construction of the adjoint mit ocean general circulation model and application to Atlantic heat transport sensitivity. J. Geophys. Res. 104, 29529-29548.

Marshall, J., Adcroft, A., Hill, C., Perelman, L., Heisey, C., 1997. A finite-volume, incompressible Navier-Stokes model for studies of the ocean on parallel computers. J. Geophys. Res. 102.

Mertz, G., Wright, D., 1992. Interpretations of the JEBAR term. J. Phys. Oceanogr. 22, 301-305.

Reynaud, T., Le Grand, P., Mercier, H., Barnier, B., 1998. A new analysis of hydrographic data in the Atlantic and its application to an inverse modeling study. Intern. WOCE News. 32, 29-31.

Roemmich, D., Boebel, D., Desaubies, Y., Freeland, H., King, B., Le Traon, P., Molinari, 
R., Owens, W., Riser, S., Send, U., Takeuchi, K., Wijffels, S., 1999. ARGO : the global array of profiling floats. OCEANOBS.

Schiller, A., Wijffels, S., Meyers, G., 2004. Design requirements for an argo float array in the indian ocean inferred from observing system simulation experiments. J. Atmos. Ocean. Techn. 21, 1598-1620.

Stammer, D., 1997. Global characteristic of ocean variability estimated from regional TOPEX/POSEIDON altimeter measurements. J. Geophys. Res. 27, 1743-1770.

Stammer, D., Wunsch, C., Giering, R., Eckert, C., Heimbach, P., Marotzke, J., Adrcoft, A., Hill, C., Marshall, J., 2002. Global ocean circulation during 1992-1997, estimated from observations and a general circulation model. J. Geophys. Res. 107.

Thacker, W., Long, R., 1988. Fitting dynamics to data. J. Geophys. Res. 93, 1227-1240.

Weaver, A., Courtier, P., 2001. Correlation modeling on the sphere using a generalized diffusion equation. Q. J. R. Meteorol. Soc. 127.

Weaver, A., Vialard, J., Anderson, D., 2003. Three- and four-dimensional variational assimilation with a general circulation model of the tropical Pacific Ocean. part 1 : formulation, internal diagnostics, and consistency checks. Month. Weath. Rev. 131, 13601378.

Wunsch, C., 1996. The Ocean Circulation Inverse Problem. Cambridge University Press. 


\section{List of Figure captions}

Figure 1 : Error profiles associated with the data (solid lines; $\left.\sigma_{o}(k)\right)$ and the initial conditions (dashed lines; $\sigma_{b}(k)$ ) in ARGOlike.

Figure 2 : Model coast line (black), model $2000 \mathrm{~m}$ isobath (grey), and simulated profile positions (crosses) over the whole year in ARGOlike.

Figure 3 : Distance to observations ( $j_{o}$ and $j_{\text {perfect }}$, as defined in Section 2.1) as a function of time, in ARGOlike. Left: average of $j_{o}^{r e s}$ (solid lines), and $j_{o}^{s i g}$ (dashed lines); for $\mathrm{T}$ (thick lines), and S (thin lines). Right: same but for $j_{\text {perfect }}$ instead of $j_{o}$.

Figure 4 : Mean squared signal (upper panels) and residual (lower panels), for T (left) and $\mathrm{S}$ (right), as a function of time and depth in ARGOlike. Values at one model level are normalized by the time averaged mean squared signal at this level. Signal and residual are errors before and after assimilation respectively (see Section 2.3). The vertical coordinate is stretched to emphasize the upper $500 \mathrm{~m}$.

Figure 5 : Signal (A) and residual (B) at $847.5 \mathrm{~m}$, for $\mathrm{T}$ (in ${ }^{\circ} \mathrm{C}$ ), averaged over the last 6 months, in ARGOlike. Lower panels: residual in fewerFLOATS $(\mathrm{C})$ and moreFLOATS (D). Lower left panel only: profile positions (crosses) over the whole year, in fewerFLOATS.

Figure 6 : Signal (A) and residual (B) at $40.3^{\circ} \mathrm{W}$ and $160 \mathrm{~m}$, for $\mathrm{T}\left(\right.$ in ${ }^{\circ} \mathrm{C}$ ), as a function of time and latitude, in ARGOlike. Lower panels: opposite of the correction resulting from the assimilation, for $\mathrm{T}(\mathrm{C})$ and $\mathrm{S}(\mathrm{D})$.

Figure 7 : Same as Figure 4 but for the zonal velocity (U; left) and for the meridional velocity (V; right).

Figure 8 : Signal (A) and residual (B) for the velocity field at $847.5 \mathrm{~m}$, averaged over the last 6 months, in ARGOlike. Velocity anomalies weaker than $0.5 \mathrm{~cm} \mathrm{~s}^{-1}$ are masked; those larger than $2 \mathrm{~cm} \mathrm{~s}^{-1}$ have thicker arrows.

Figure 9 : Signal (left) and residual (right) for the sea surface height, averaged over the last 6 months, in ARGOlike. Contour interval: $0.04 \mathrm{~m}$. Negative and positive values are shown as grey and black contours respectively.

Figure 10 : Signal (left) and residual (right) for the barotropic streamfunction (upper; Eq. 2) and the overturning streamfunction (lower; Eq. 3), averaged over the last 6 months, in ARGOlike. Contour intervals: $4 \mathrm{~Sv}$ and $1 \mathrm{~Sv}$ respectively $\left(1 \mathrm{~Sv}=10^{6} \mathrm{~m}^{3} \mathrm{~s}^{-1}\right)$.

Figure 11 : Signal (solid lines) and residual (solid lines with circles) for the advective heat flux $\left(\Theta ;\right.$ Eq. 4), its overturning component $\left(\Theta_{o v} ;\right.$ Eq. 5), and its gyre component $\left(\Theta_{g y} ;\right.$ Eq. 6), averaged over the last 6 months, in ARGOlike. Also shown: residual in exp. moreFLOATS (dashed lines). Unit: PW $\left(1 \mathrm{PW}=10^{15} \mathrm{~W}\right)$. 
Table 1

Reserved terms throughout the discussion of experiments.

\begin{tabular}{|ll|}
\hline Signal & $=$ first guess - reference \\
\hline Residual & $=$ optimal - reference \\
\hline Correction & $=$ optimal - first guess \\
\hline
\end{tabular}

Table 2

Summary of the observing systems' characteristics. N/S is a noise to signal ratio, defined in Section 2.4. All experiments marked with $\left(^{*}\right)$ start from the same model solution.

\begin{tabular}{|l||c|c|c|c|}
\hline Experiment & number of profilers & array type & $N / S$ for T & $N / S$ for S \\
\hline \hline ARGOlike $\left(^{*}\right)$ & 300 & drifting & 1.4 & 1.5 \\
\hline \hline moreNOISE $\left(^{*}\right)$ & 300 & drifting & 2.5 & 2.5 \\
\hline lessNOISE $(*)$ & 300 & drifting & 0.25 & 0.25 \\
\hline moreFLOATS $\left.*^{*}\right)$ & 900 & drifting & 1.4 & 1.5 \\
\hline fewerFLOATS $\left.*^{*}\right)$ & 100 & drifting & 1.4 & 1.5 \\
\hline RANDOM $(*)$ & 300 & see Section 4$)$ & 1.4 & 1.5 \\
\hline EULERIAN $\left.*^{*}\right)$ & 300 & moored & 1.4 & 1.5 \\
\hline \hline signal1 & 300 & drifting & 1.7 & 1.8 \\
\hline signal2 & 300 & drifting & 1.5 & 1.6 \\
\hline signal3 & 300 & drifting & 1.9 & 2.0 \\
\hline
\end{tabular}


Table 3

Mean squared residual (errors after assimilation) expressed as a percentage of mean squared signal (errors before assimilation), for the last 6 months, for the observed model levels (between 0 and $2450 \mathrm{~m}$ ). Mean squared residual and signal are first computed at each vertical level and for each month. Next, they are averaged over the set of months. Then the ratio of mean squared residual to mean squared signal is computed. Finally, the result is averaged over the set of model levels.

\begin{tabular}{|l||c|c|c|c|}
\hline Experiment & $\mathrm{T}$ & $\mathrm{S}$ & $\mathrm{U}$ & $\mathrm{V}$ \\
\hline \hline ARGOlike & 26 & 35 & 30 & 36 \\
\hline moreNOISE & 33 & 42 & 38 & 42 \\
\hline lessNOISE & 13 & 21 & 14 & 20 \\
\hline moreFLOATS & 10 & 15 & 13 & 16 \\
\hline fewerFLOATS & 42 & 56 & 47 & 51 \\
\hline RANDOM & 13 & 17 & 18 & 18 \\
\hline EULERIAN & 22 & 31 & 27 & 30 \\
\hline
\end{tabular}

Table 4

Same as Table 3 but for different realizations of the signal.

\begin{tabular}{|l||c|c|c|c|}
\hline Experiment & $\mathrm{T}$ & $\mathrm{S}$ & $\mathrm{U}$ & $\mathrm{V}$ \\
\hline \hline ARGOlike & 26 & 35 & 30 & 36 \\
\hline moreNOISE & 33 & 42 & 38 & 42 \\
\hline signal1 & 34 & 39 & 35 & 39 \\
\hline signal2 & 20 & 30 & 30 & 38 \\
\hline signal3 & 32 & 33 & 35 & 45 \\
\hline
\end{tabular}


Fig. 1. Error profiles associated with the data (solid lines; $\sigma_{o}(k)$ ) and the initial conditions (dashed lines; $\left.\sigma_{b}(k)\right)$ in ARGOlike.
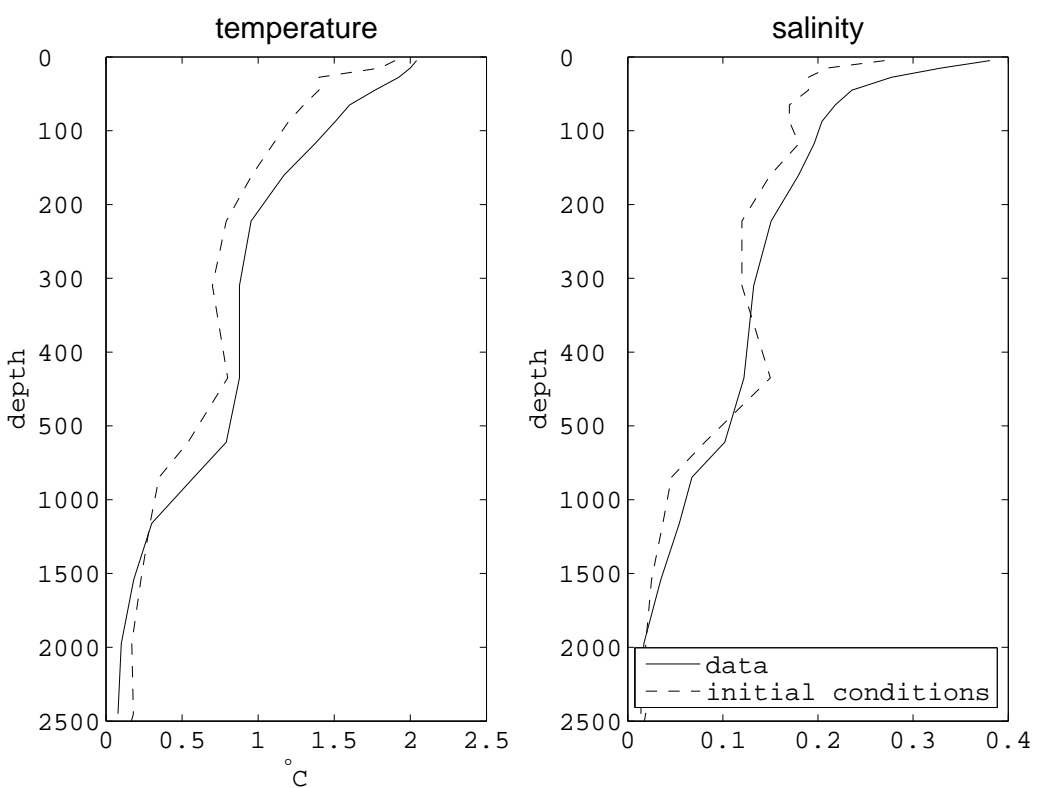

Fig. 2. Model coast line (black), model $2000 \mathrm{~m}$ isobath (grey), and simulated profile positions (crosses) over the whole year in ARGOlike.

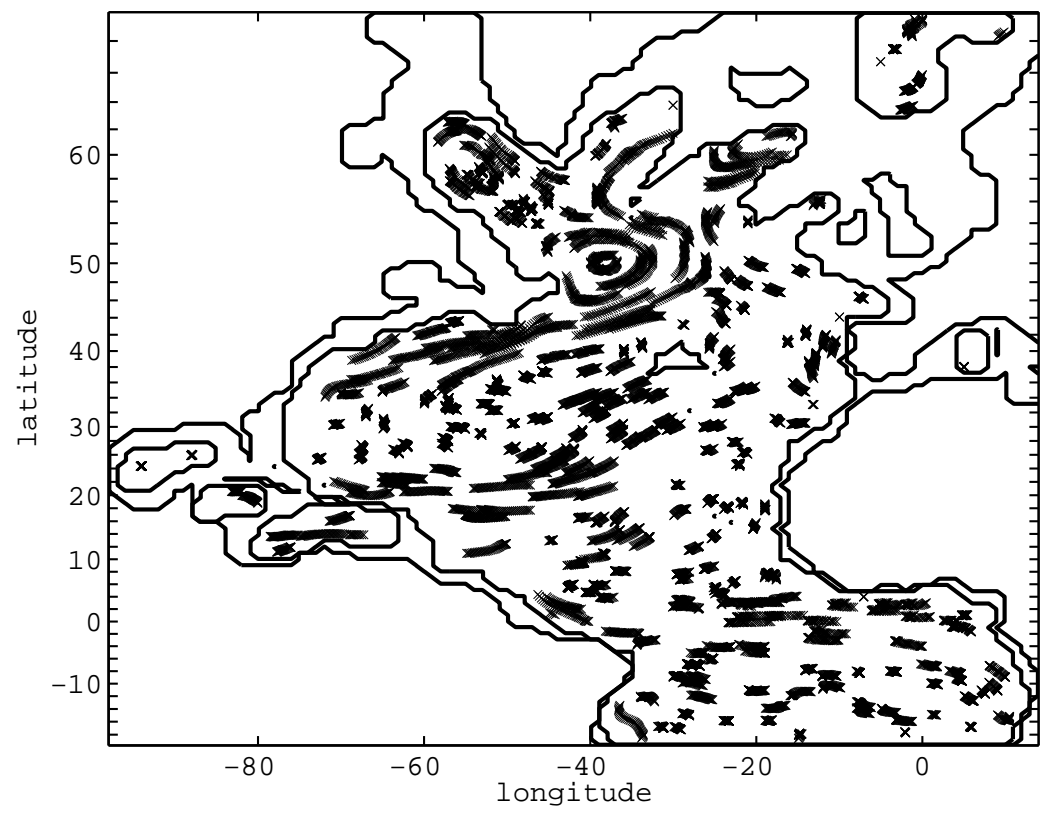


Fig. 3. Distance to observations ( $j_{o}$ and $j_{\text {perfect }}$, as defined in Section 2.1) as a function of time, in ARGOlike. Left: average of $j_{o}^{\text {res }}$ (solid lines), and $j_{o}^{\text {sig }}$ (dashed lines); for $\mathrm{T}$ (thick lines), and $\mathrm{S}$ (thin lines). Right: same but for $j_{\text {perfect }}$ instead of $j_{o}$.
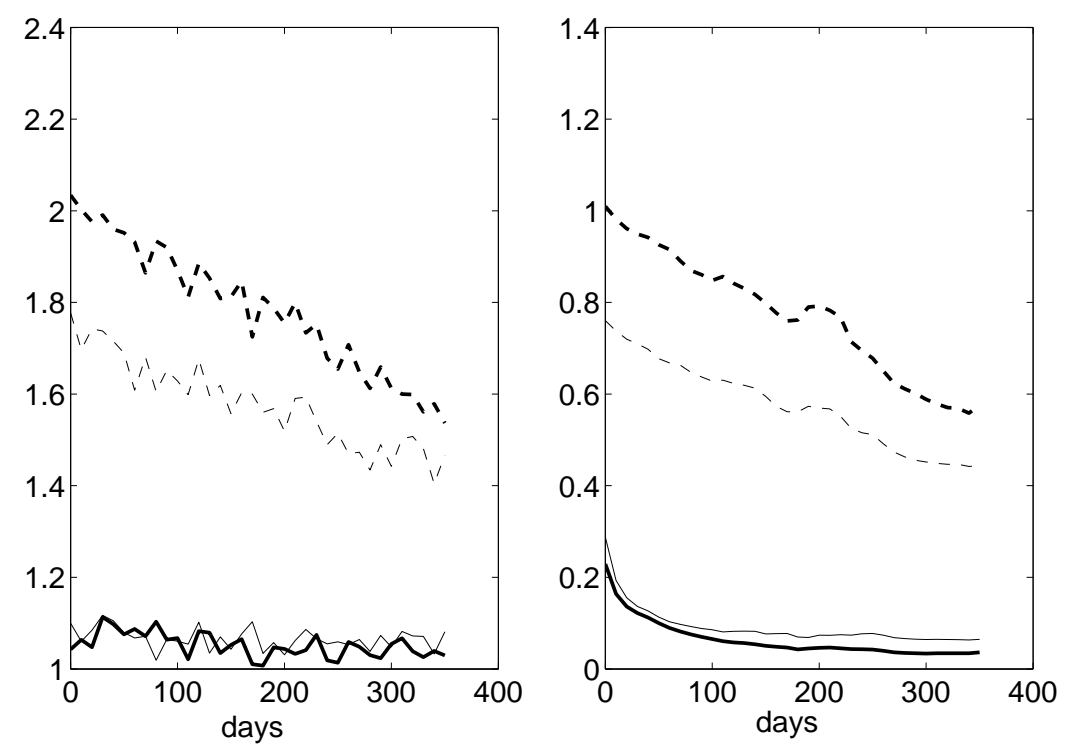
Fig. 4. Mean squared signal (upper panels) and residual (lower panels), for T (left) and S (right), as a function of time and depth in ARGOlike. Values at one model level are normalized by the time averaged mean squared signal at this level. Signal and residual are errors before and after assimilation respectively (see Section 2.3). The vertical coordinate is stretched to emphasize the upper $500 \mathrm{~m}$.
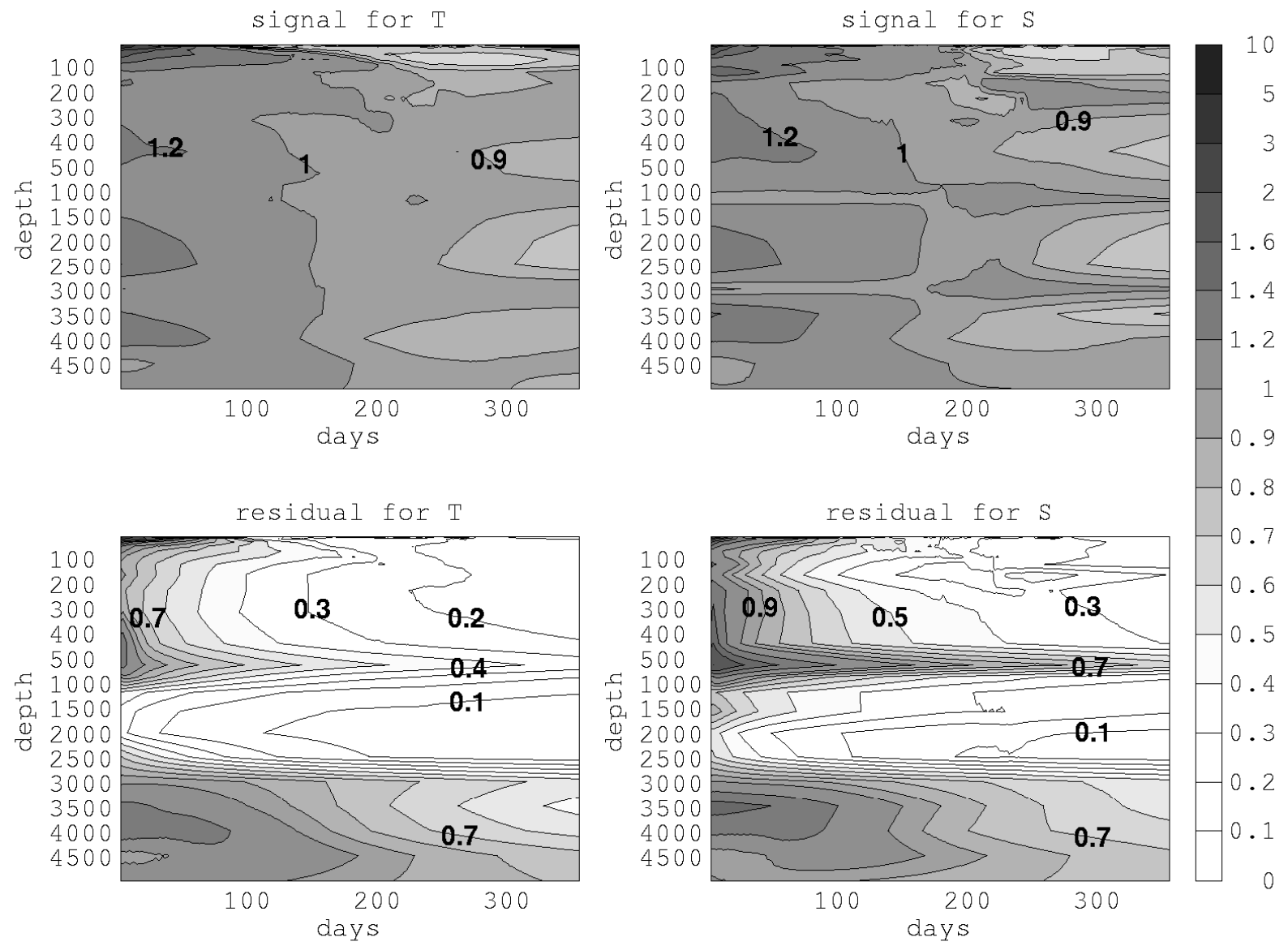
Fig. 5. Signal (A) and residual (B) at $847.5 \mathrm{~m}$, for $\mathrm{T}$ (in ${ }^{\circ} \mathrm{C}$ ), averaged over the last 6 months, in ARGOlike. Lower panels: residual in fewerFLOATS (C) and moreFLOATS (D). Lower left panel only: profile positions (crosses) over the whole year, in fewerFLOATS.
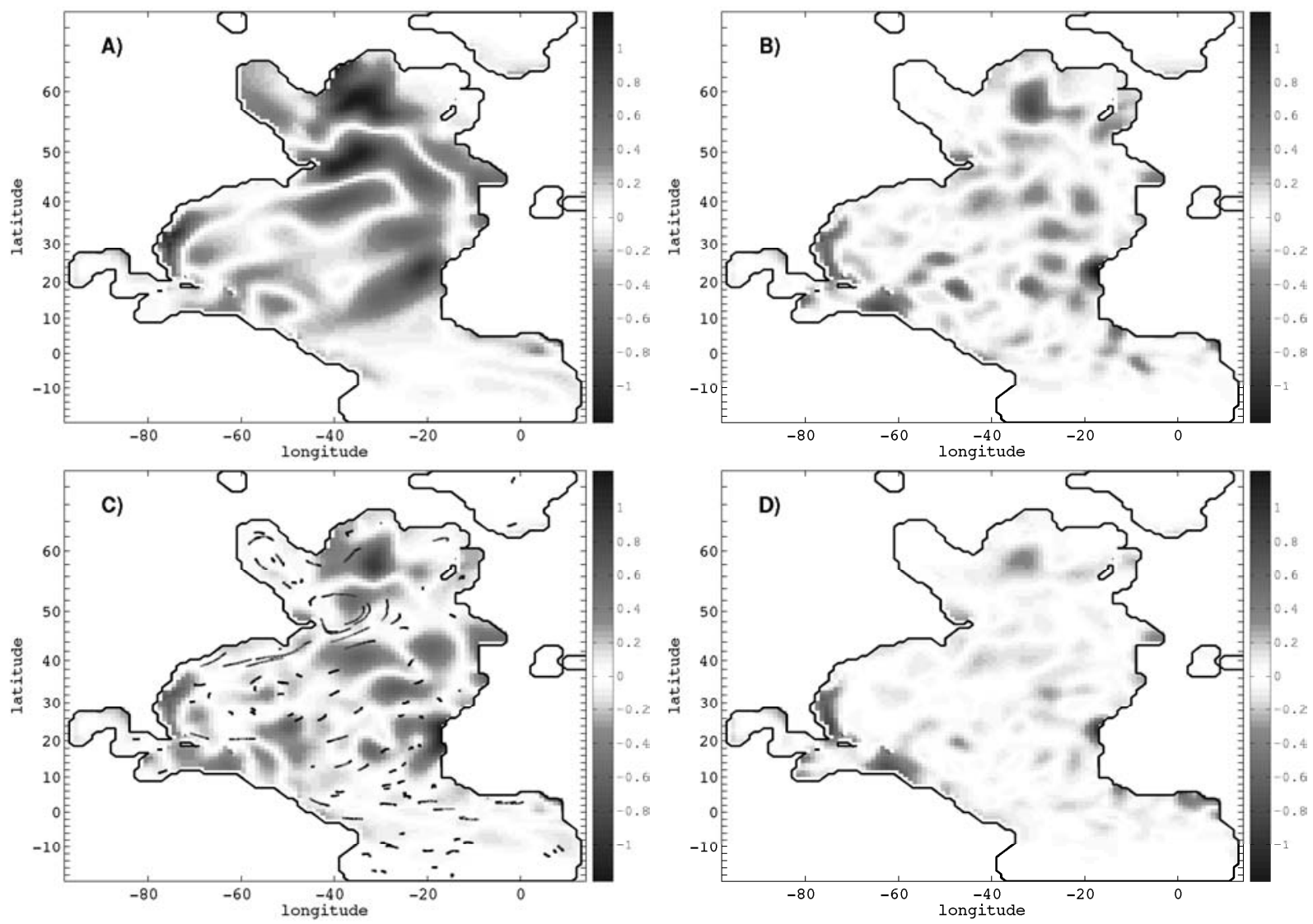
Fig. 6. Signal (A) and residual (B) at $40.3^{\circ} \mathrm{W}$ and $160 \mathrm{~m}$, for $\mathrm{T}\left(\right.$ in ${ }^{\circ} \mathrm{C}$ ), as a function of time and latitude, in ARGOlike. Lower panels: opposite of the correction resulting from the assimilation, for $\mathrm{T}(\mathrm{C})$ and $\mathrm{S}(\mathrm{D})$.
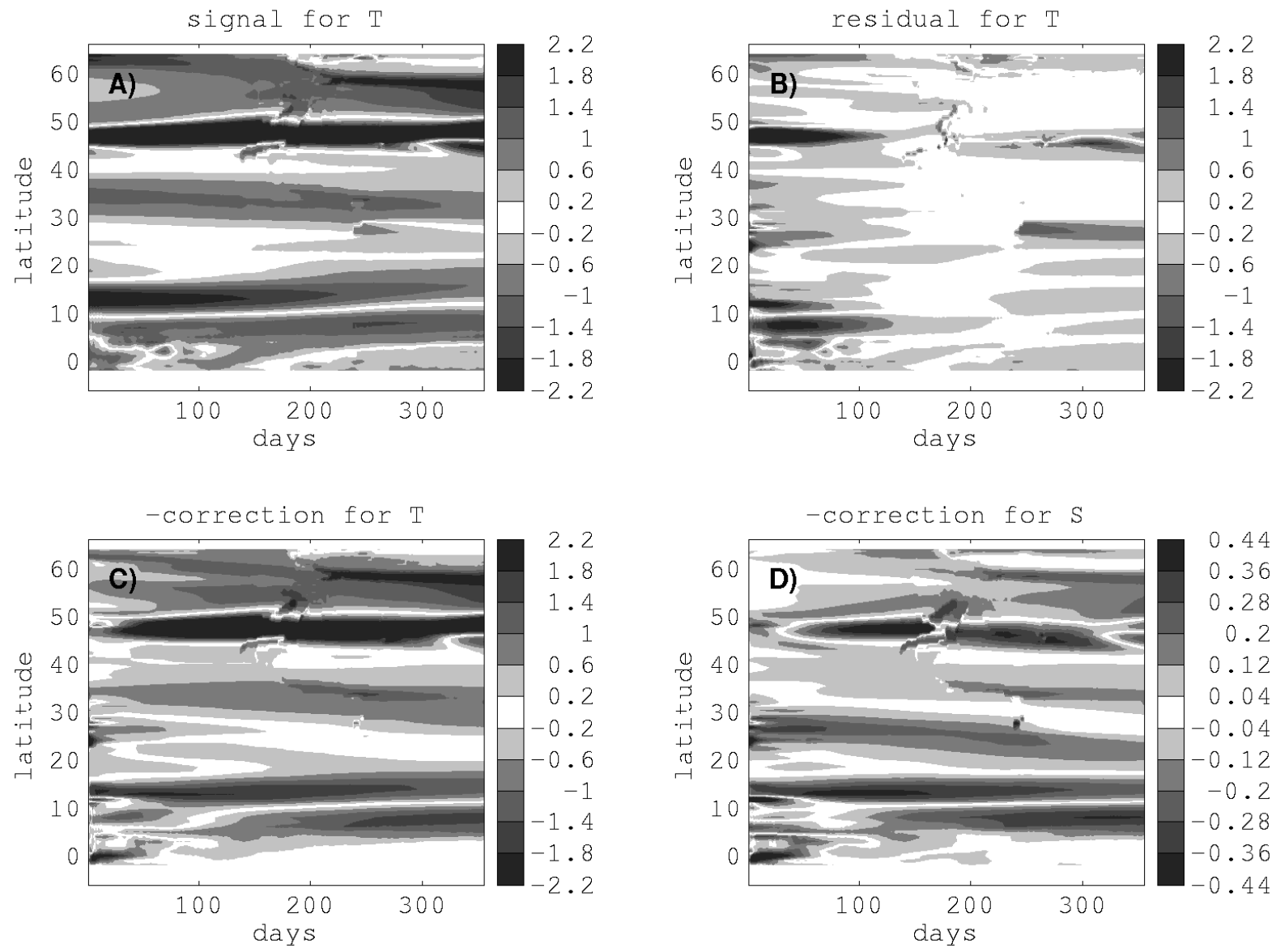
Fig. 7. Same as Figure 4 but for the zonal velocity (U; left) and for the meridional velocity (V; right).
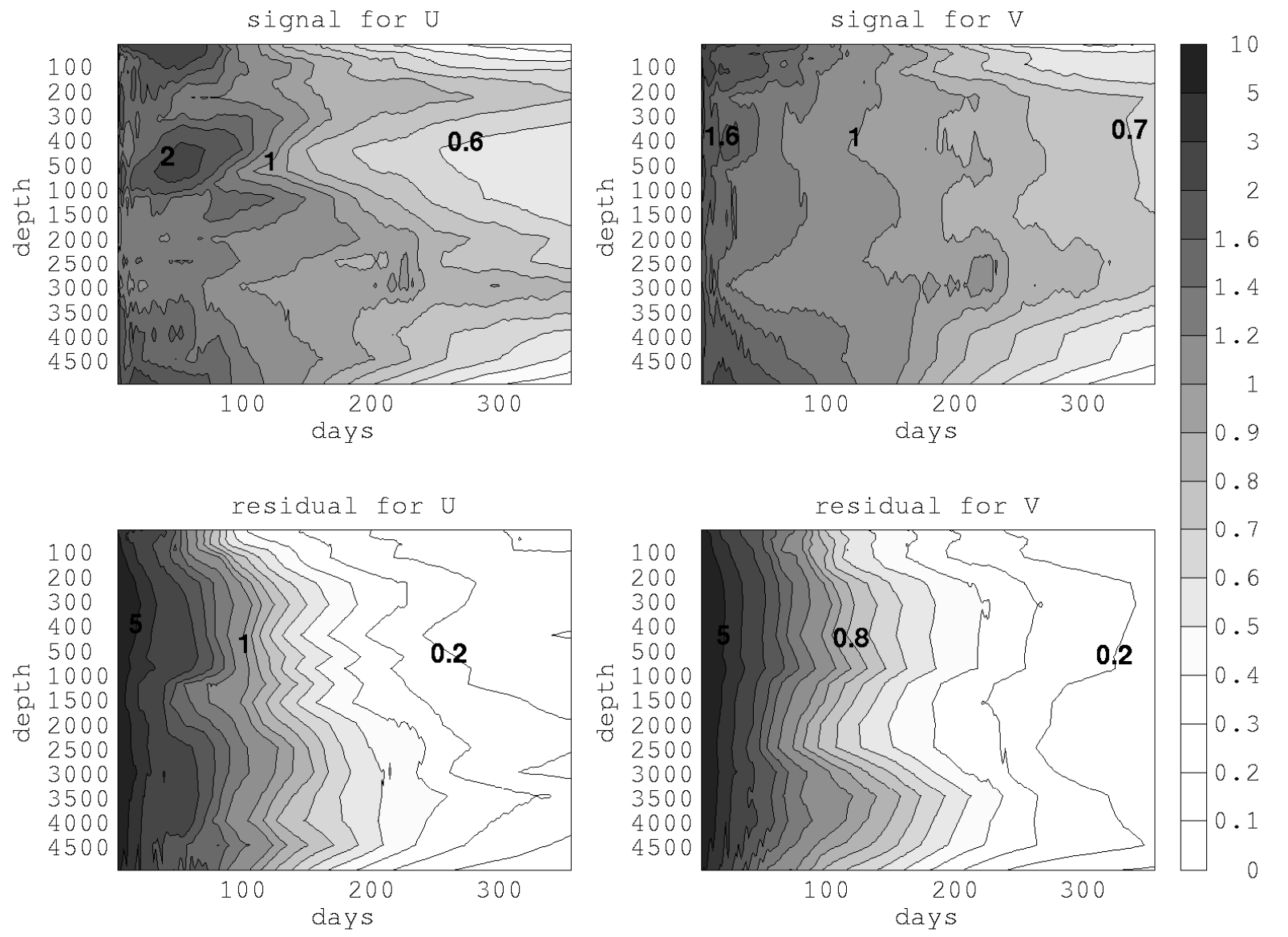
Fig. 8. Signal (A) and residual (B) for the velocity field at $847.5 \mathrm{~m}$, averaged over the last 6 months, in ARGOlike. Velocity anomalies weaker than $0.5 \mathrm{~cm} \mathrm{~s}^{-1}$ are masked; those larger than $2 \mathrm{~cm} \mathrm{~s}^{-1}$ have thicker arrows.
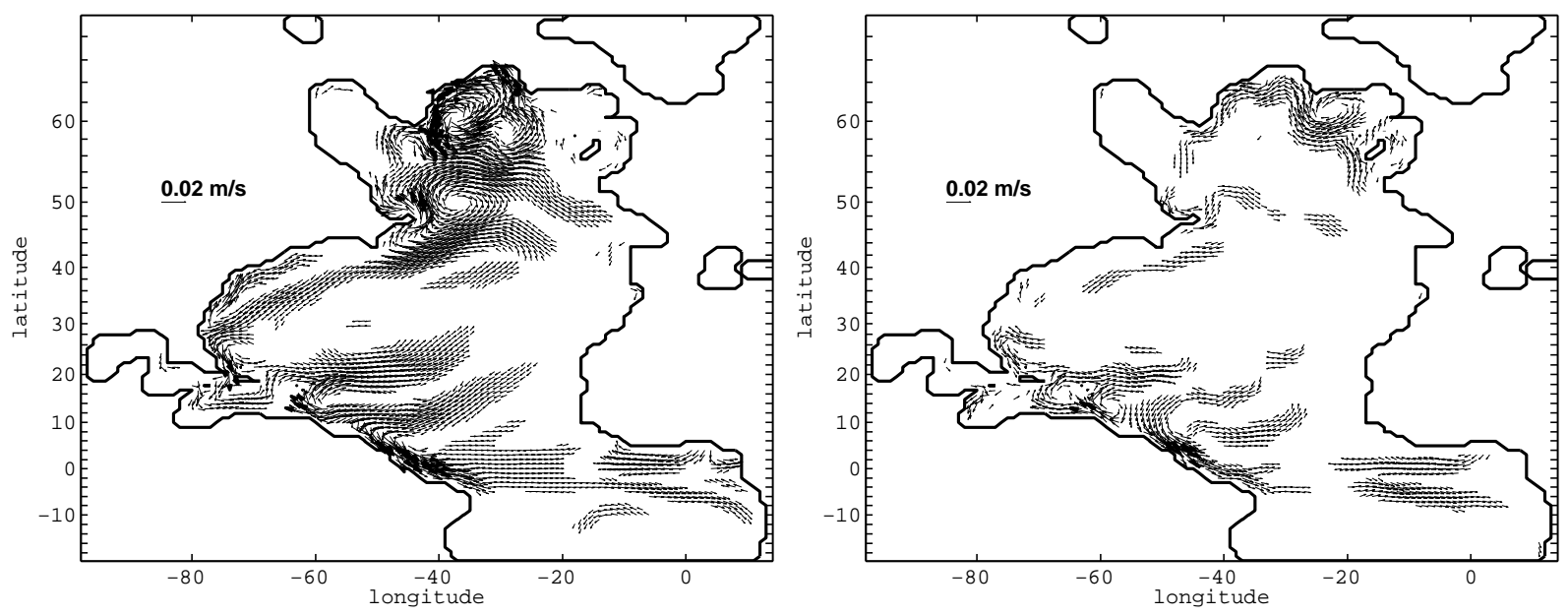

Fig. 9. Signal (left) and residual (right) for the sea surface height, averaged over the last 6 months, in ARGOlike. Contour interval: $0.04 \mathrm{~m}$. Negative and positive values are shown as grey and black contours respectively.
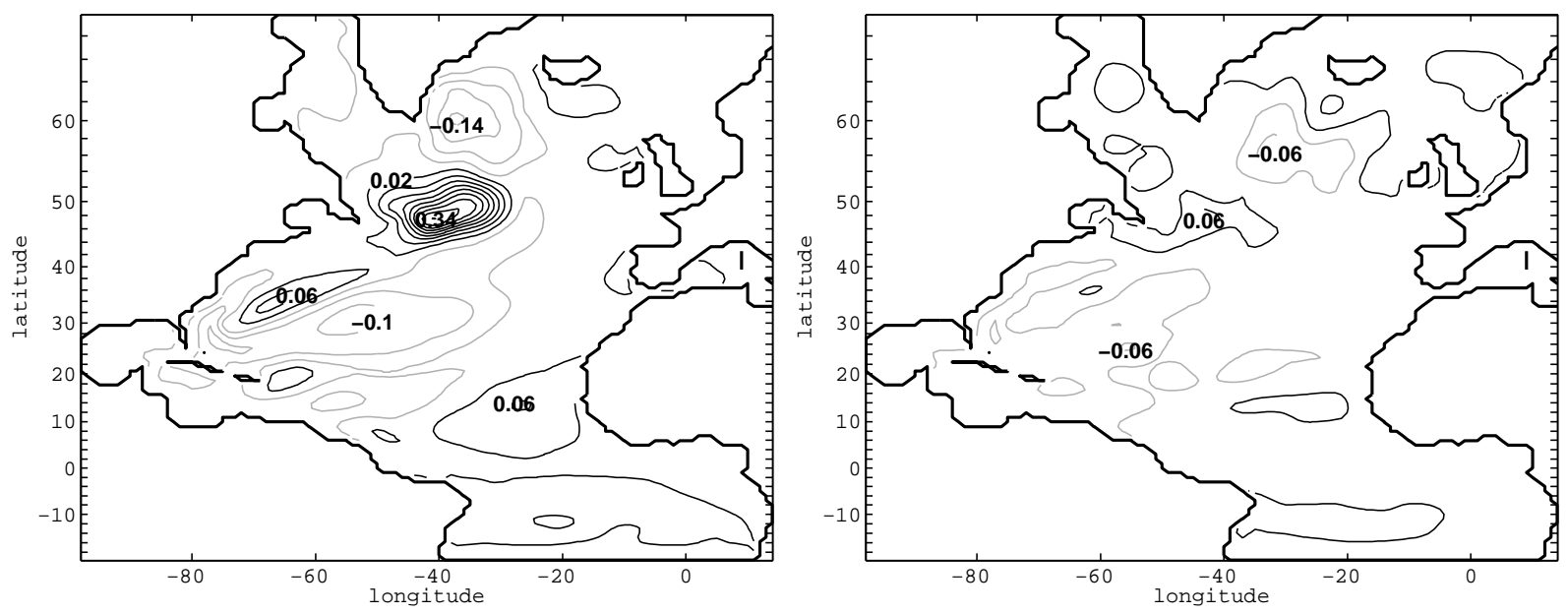
Fig. 10. Signal (left) and residual (right) for the barotropic streamfunction (upper; Eq. 2) and the overturning streamfunction (lower; Eq. 3), averaged over the last 6 months, in ARGOlike. Contour intervals: $4 \mathrm{~Sv}$ and $1 \mathrm{~Sv}$ respectively $\left(1 \mathrm{~Sv}=10^{6} \mathrm{~m}^{3} \mathrm{~s}^{-1}\right)$.
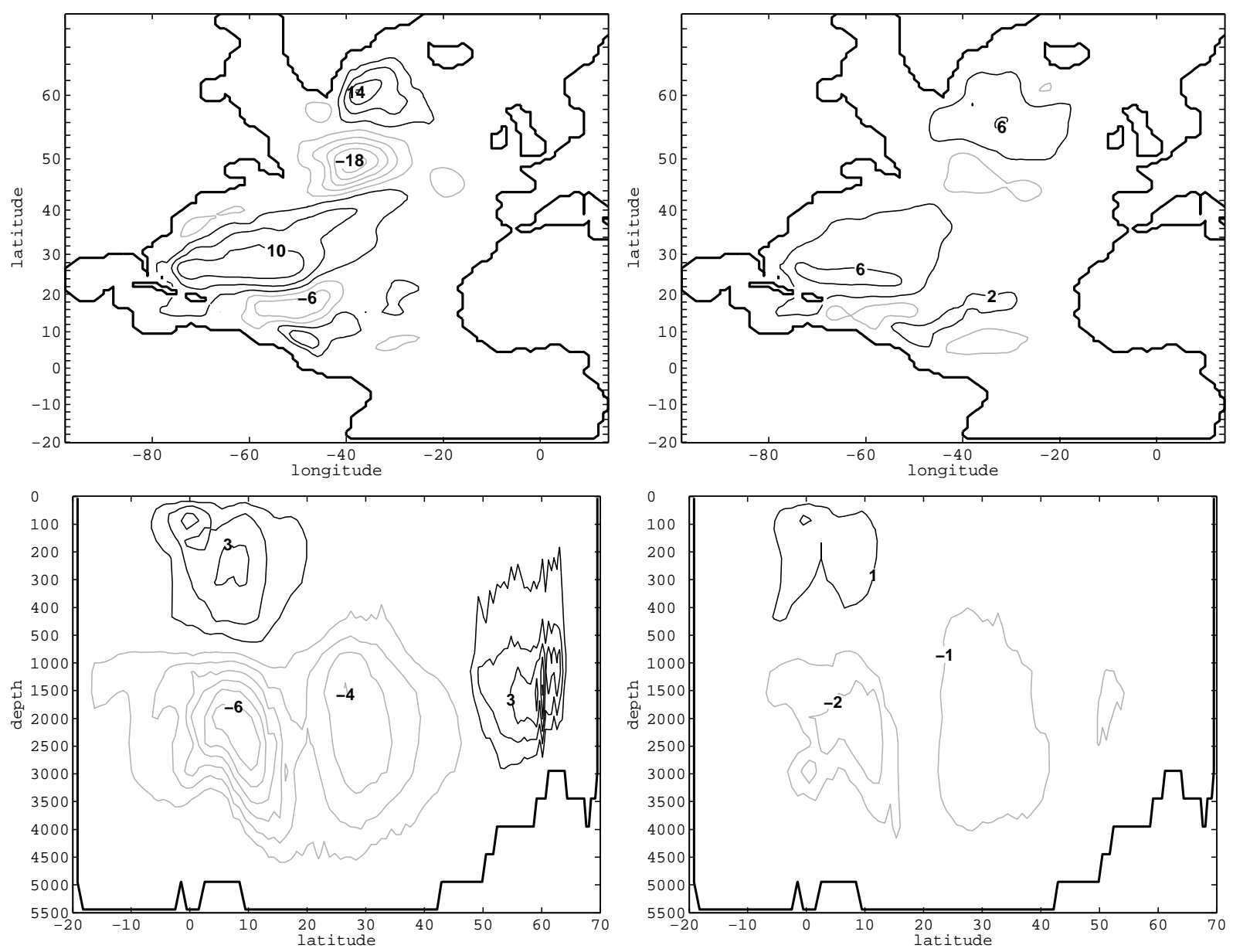
Fig. 11. Signal (solid lines) and residual (solid lines with circles) for the advective heat flux $(\Theta$; Eq. 4), its overturning component $\left(\Theta_{o v}\right.$; Eq. 5), and its gyre component $\left(\Theta_{g y} ;\right.$ Eq. 6), averaged over the last 6 months, in ARGOlike. Also shown: residual in exp. moreFLOATS (dashed lines). Unit: PW (1 PW = $\left.10^{15} \mathrm{~W}\right)$.
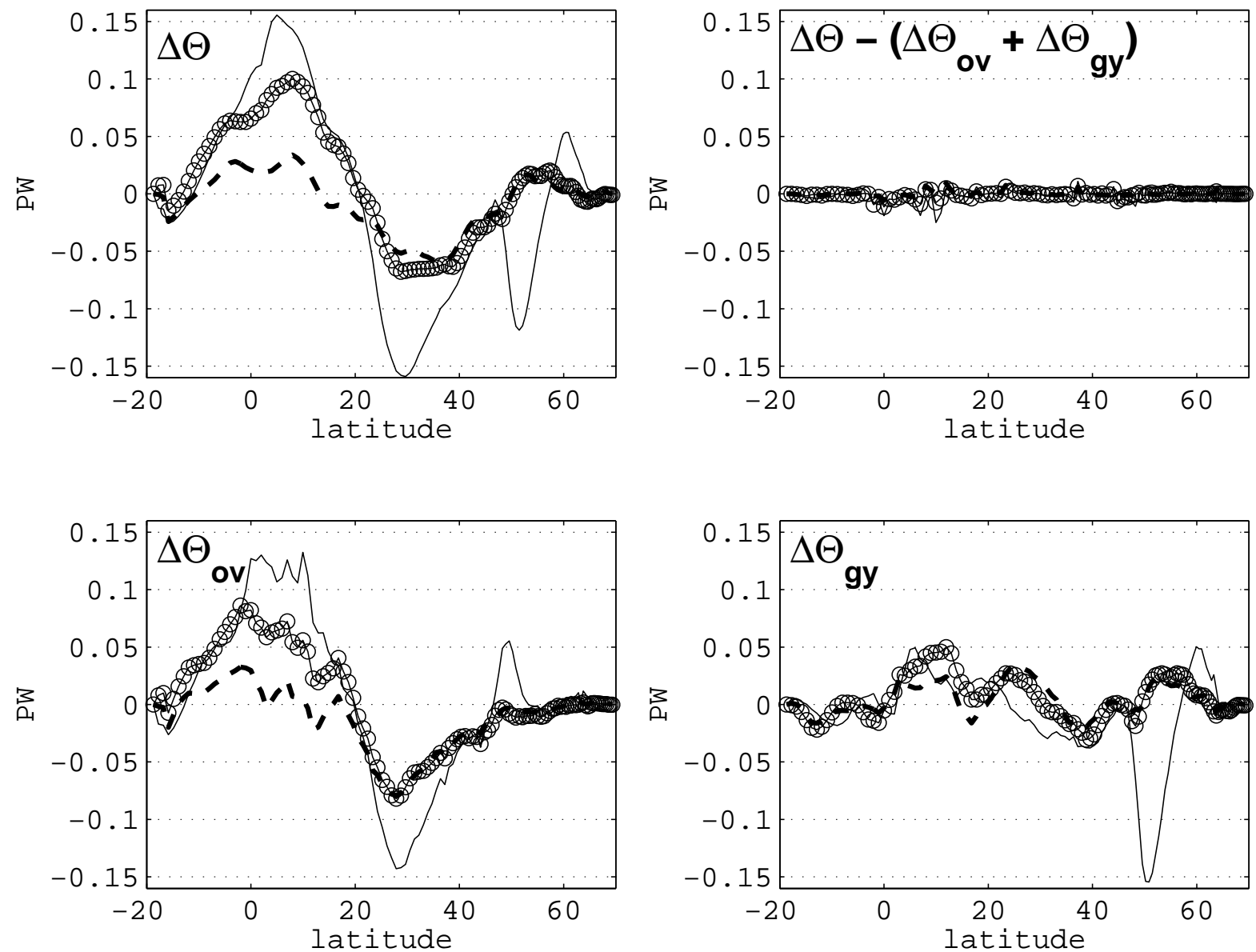